Journal of Bangladesh College of Physicians and Surgeons

Vol. 28, No. 3, September 2010

\title{
Evaluation \& Management of Obscure Gastrointestinal Bleeding (OGIB)
}

\author{
S PERVEEN ${ }^{\mathrm{a}}$, MR HOSSAIN $^{\mathrm{b}}, \mathrm{SMB}^{\mathrm{H}}$ HUSSAIN $^{\mathrm{c}}$, MA AHMED $^{\mathrm{d}}, \mathrm{H} \mathrm{AFTAB}^{\mathrm{e}}$
}

\begin{abstract}
Summary:
Gastrointestinal bleeding is a common entity. Incidence of bleeding has comparatively increased though case fatality is static. Despite improved treatments and better understanding of the underlying pathophysiology of peptic ulcer disease the rising figures of GI bleeding reflect an increasing proportion of elderly population and nonsteroidal anti-inflammatory use. Overall, $5 \%$ of all cases of gastrointestinal bleeding fall under the category of Obscure gastrointestinal bleeding (OGIB) in the USA. Obscure gastrointestinal bleeding is defined as bleeding of unknown origin that persists or recurs after an initial negative
\end{abstract}

\section{Introduction:}

Bleeding from the upper gastrointestinal (GI) tract remains common, with a reported annual incidence of up to 172 per $100000^{1}$, which has increased. Case fatality was recently reported as $14 \%{ }^{2}$ which is static, despite improved treatments and better understanding of the underlying pathophysiology of peptic ulcer disease. The rising figures may reflect an increasing proportion of elderly patients and non-steroidal antiinflammatory use. Of patients in whom a diagnosis is confirmed, more than $90 \%$ suffer from peptic ulcers, oesophageal or gastric malignancy, varices, MalloryWeiss syndrome, erosive disease and oesophagitis ${ }^{1,2}$. Less common aetiologies of upper GI bleeding which are sometimes difficult to identify at endoscopy and manage will be the focus of this study.

\section{Definition}

Obscure gastrointestinal bleeding (OGIB) is defined as bleeding of unknown origin that persists or recurs after an initial negative endoscopic evaluation including

a. Lt Col Shaila Perveen, MBBS, MCPS, FCPS.

b. Maj Gen Muhammad Rabiul Hossain, MBBS, MCPS, FCPS, FRCP

c. Brig Gen Sk. Md. Bahar Hussain, MBBS, FCPS, FRCP

d. Lt Col Mir Azimuddin Ahmed, MBBS, DCP, MCPS, FCPS

e. Dr. Hafeza Aftab, MBBS, MD

Address of Correspondence: Dr. Shaila Perveen, Classified Medical Specialist, CMH Patenga, Chittagong, Phone: 01819294922

Received: 10 December, 2009

Accepted: 28 June, 2010 endoscopic evaluation including colonoscopy and/or upper endoscopy. OGIB can be either Occult (no visible blood) or Overt (Passage of visible blood).

Less common aetiologies of GI bleeding e.g. Cameron erosions, Dieulafoy's lesion, Watermelon stomach that are sometimes difficult to identify at endoscopy often present as OGIB. They need special techniques even thrombolytic therapy to precipitate bleeding for diagnostic angiography. Increased awareness of the existence of such conditions help in rapid and accurate identification of the lesion. Review of such cases will be the focus of this publication.

(J Bangladesh Coll Phys Surg 2010; 28: 174-182)

colonoscopy and/or upper endoscopy (esophagogastroduodenoscopy [EGD]). ${ }^{3}$ OGIB can be classified as either:

(1) Occult OGIB- which is manifested by recurrent iron deficiency anemia and/or recurrent positive fecal occult blood test (FOBT) results

(2) Overt OGIB- which is manifested as melena or hematochezia.

Overall, OGIB accounts for 5\% of all cases of gastrointestinal bleeding in the USA ${ }^{4}$. Angiectasias of the small bowel are the most common source of OGIB and account for $30 \%$ to $40 \%$ of gastrointestinal bleeding in the elderly population, whereas tumors such as leiomyomas, carcinoids, lymphomas and adenocarcinomas are the predominant cause in patients aged 30 to 50 years ${ }^{5}$. Meckel's diverticulum, erosions and ulcers from nonsteroidal anti-inflammatory drug (NSAID) use ${ }^{6}$ and Crohn's disease of small bowel are also potential causes of OGIB. The term 'midgastrointestinal bleeding' rather than obscure bleeding is now applied if the origin is thought to be between papilla and ileocaecal valve ${ }^{7}$.

\section{Causes of Obscure GI Blood Loss:}

Common causes (any site):

Peptic ulcer, Reflux esophagitis, Erosive gastritis, Carcinoma(Specially colon), Vascular ecstasia / angiodysplasia, Chron's disease 


\section{Uncommon Causes (Upper GIT)}

1. Esophagus / Stomach

Dieulafoy's lesion, Cameron's erosions with hiatal hernia, Prolapse erosions,

Gastric antral vascular ecstasia (Water melon stomach), Portal gastropathy, Varices

2. Small Intestine

Meckel's diverticulum, Celiac sprue, Chron's disease, Duodenitis

\section{Uncommon Causes (Lower GIT)}

3. Colon

Diverticula (obscure overt bleeding), Colitis (ulcerative/ ischaemic/ radiation injury), Endometriosis, Infection (hookworm, ascariasis, whipworm, strongiloidosis, amoebiasis, cytomegalovirus, tubercular entero colitis)

4. Rectum

Fissure, Haemorrhoid

5. Any site

Vasculitis, Telengiectasia, Aorto enteric fistula, Other cancers (lymphoma-gastric NHL, Kaposi's, leiomyoma, sarcoma, melanoma, carcinoids), Large polyps, Blue rubber bleb nevus syndrome, Haemangioma, Radiation damage, Amyloidosis

6. Extra intestinal

Haemobilia, Wirsungorrhoea, Haemoptysis, Epistaxis, bleeding gums

7. No source identified.

\section{Angiodysplasia}

Gastrointestinal angiodysplasias are the most common cause of obscure chronic blood loss from the digestive tract with small bowel angiodysplasia accounting for up to $40 \%$ of obscure GI bleeding ${ }^{8}$. The pathophysiology is unknown, but has been suggested to result from low grade venous obstruction of submucosal veins as they cross muscle layers ${ }^{[9]}$. It is said to be more prevalent in chronic renal failure patients ${ }^{[10]}$ and in patients with aortic stenosis, although, recent reports have failed to confirm this link ${ }^{11}$. Osler-WeberRendu Syndrome is an autosomal dominant condition characterized by angiodysplastic lesions involving the skin, mucosal membranes and organs other than the GI tract (Figure 1). Their endoscopic appearance is indistinguishable from other angiodysplastic lesions (Figure 2) but more widespread.

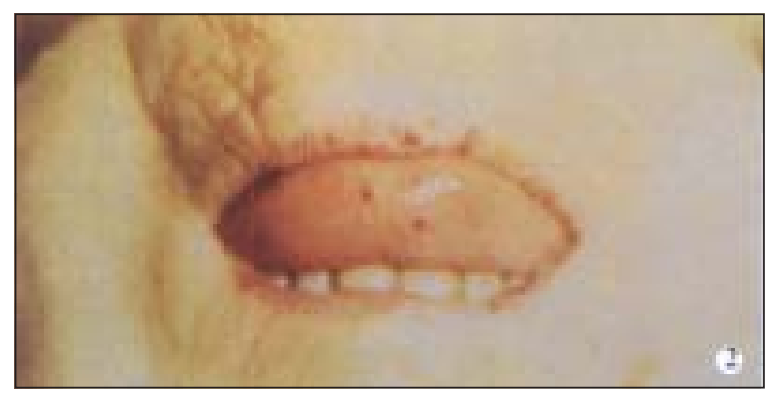

Fig.-1: Cutaneous manifest of $O-W-R$

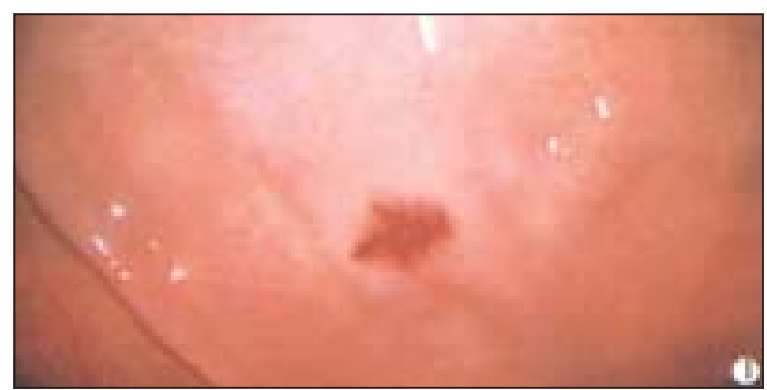

Fig.-2: Mucosal lesion of OWR

\section{Dieulafoy's Lesion}

Dieulafoy's lesion is a cause of diagnostic difficulty in patients with repeated haematemesis. The exposed, eroded vessel in a very small mucosal defect is difficult to spot at endoscopy (Figure 3) and accounts for perhaps $2 \%$ of upper GI bleeds ${ }^{12}$. It was described in detail by Dieulafoy ${ }^{13}$ in 1896 who termed 'Exulceratio Simplex' as bleeding from a simple tortuous abberant submucosal artery of small size. The typical endoscopic appearance is that of a dark red 'nipple'.

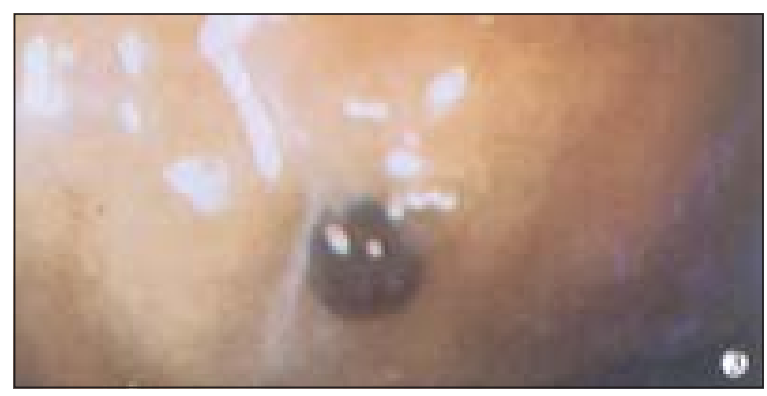

Fig.-3: Dieulafoy’s lesion 


\section{Gastric Antral Vascular Ectasia (Watermelon Stomach)}

This was first described in 1952 by Rider et al and used to be called gastric antral vascular ectasia. Jabbari et al ${ }^{[14]}$ coined the phrase "watermelon stomach" to describe the endoscopic features (Figure 4). Recognition of this characteristic lesion is important since it is commonly dismissed by less experienced endoscopists as antral gastritis.

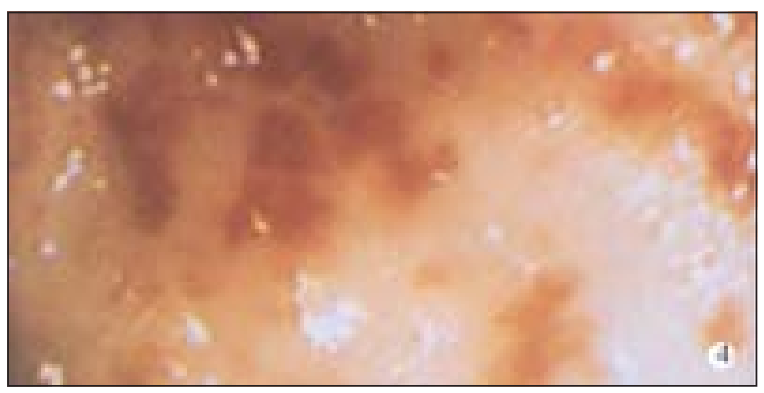

Fig.-4: Watermelon stomach

\section{Cameron Erosions}

Erosive disease is an uncommon cause of severe upper GI bleeding. However, some lesions warrant mentioning as they are often overlooked or missed at endoscopy. Cameron erosions are chronic linear erosions (Figure 5) positioned on the crests of folds at the diaphragmatic impression with a large hiatus hernia ${ }^{15}$.

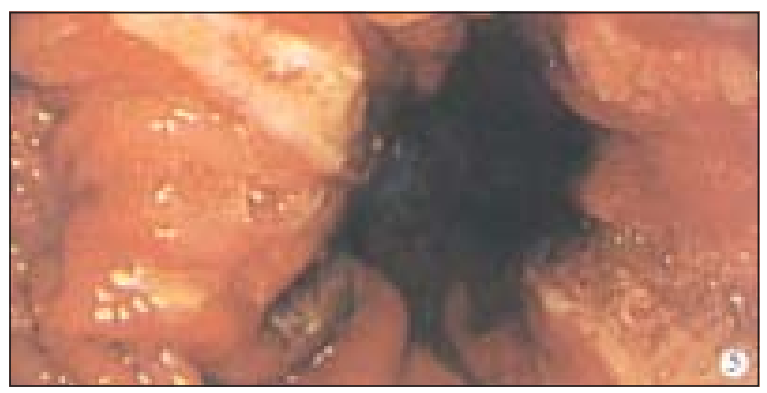

Fig.-5: Cameron lesion

\section{Prolapse Erosions}

Prolapsing gastropathy is a focal area with subepithelial haemorrhage and occasionally, erosions within a few centimeters of the cardioesophageal junction. This mucosal area prolapses into the distal oesophagus commonly from 10 o'clock position during retching, often prior to haematemesis.

\section{Unusual Upper GI Malignancies}

Adenocarcinoma accounts for $90 \%$ of gastric tumours with lymphoma accounting for $5 \%$, stromal tumours $2 \%$ and the rest include carcinoids, metastases and others. GI involvement occurs in $50 \%$ of non Hodgkins lymphoma, with the stomach being the most common extranodal site. 95\% of gastric lymphomas are nonHodgkins lymphoma.

\section{Other Vascular Disorders}

The Blue Rubber Bleb Naevus Syndrome (Figure 6) is an example of intestinal haemangioma which is an autosomal dominant condition causing GI bleeding in infants and children.

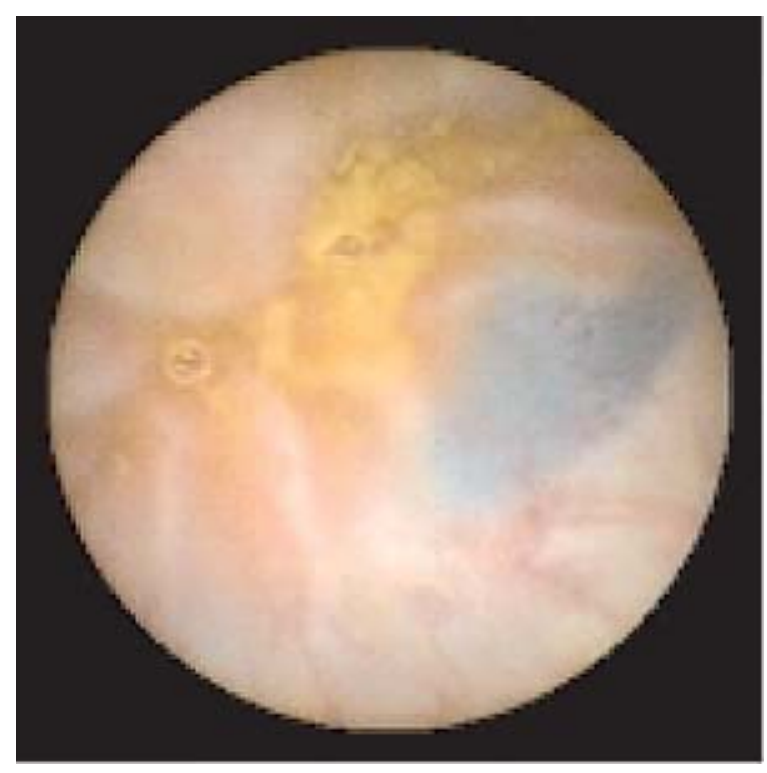

Fig.-6: Jejunal phlebectasia

\section{Haemobilia}

Bleeding from either the biliary tree (haemobilia) or from the pancreatic duct (Wirsungorrhagia) into the duodenum can be difficult to identify. Recent series indicate iatrogenic trauma accounting for $40 \%$ and accidental trauma $20 \%{ }^{16}$. Classically, patients present with the triad of pain, jaundice and melaena. A history of chronic pancreatitis or pseudocyst may be a pointer to a bleed from the pancreatic duct.

\section{Evaluation}

Evaluation starts from elaborate history and careful bedside examination to provide clue to the cause of bleeding. 


\section{History}

A history can reveal ingestion of medications known to cause bleeding (e.g., aspirin, nonsteroidal antiinflammatory drugs, alendronate, potassium chloride, anticoagulants). A family history might suggest a hereditary vascular problem.

\section{Physical Exam}

The bedside examination may be helpful in providing clues to the cause of bleeding (Table I). Rare causes of bleeding may be detected on physical examination like Plummer-Vinson syndrome, acquired immunodeficiency syndrome (AIDS), neurofibromatosis, Osler-Weber-Rendu syndrome, pseudoxanthoma elasticum, amyloidosis (Figure 7) and other diseases with typical cutaneous manifestations ${ }^{17-18}$. Symptoms specific to the upper or lower intestinal tract may direct the initial endoscopic procedure, but data do not support limiting the evaluation to the symptomatic region.

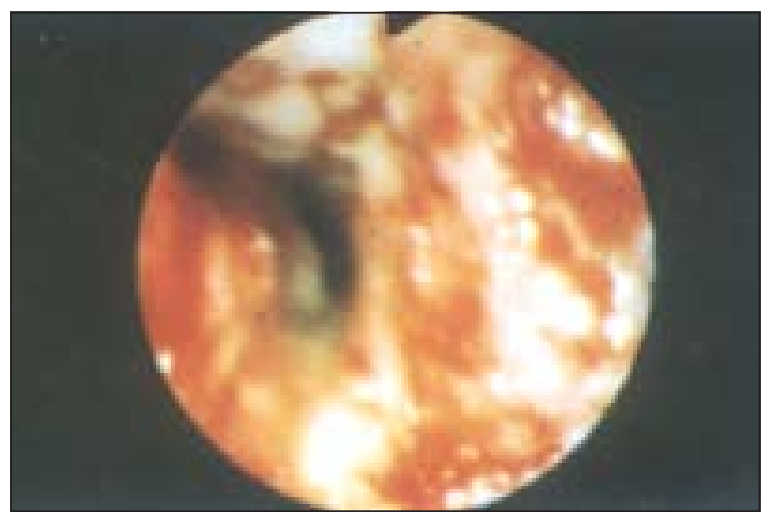

Fig.-7: Intestinal amyloid

\section{Investigations}

In OGIB, repeat EGD and colonoscopy with ileoscopy should be considered before performing a small bowel evaluation. A repeat EGD may yield a source even when the initial exam was negative. Zaman and colleagues reported that $64 \%$ of lesions identified with push enteroscopy were within reach of a standard endoscope ${ }^{19}$. Commonly missed lesions in the upper gastrointestinal tract include peptic ulcers, Cameron ulcers associated with large hiatal hernia, and angiectasias. Lesions often missed in the colon include angiectasias and neoplasms. The diagnostic yield of repeat EGD is sufficient to recommend a second-look endoscopy $20,21,22$. Adequate inflation to distend the folds in the upper stomach, a retroflexed endoscope and close examination of the mucosa posteriorly on the lesser curve may help to identify Dieulafoy's. Multiple examinations are commonly required and the abnormality is sometimes diagnosed when pulsatile arterial bleeding is seen coming from apparently normal mucosa. In the absence of clear evidence of gastrointestinal bleeding, small-bowel biopsies should be taken to rule out celiac sprue in the evaluation of patients with iron deficiency anemia ${ }^{23,24,25}$.

Gastrointestinal investigative techniques for occult and obscure bleeding are summarized in table II.

Table-I

Clinical Clues for Specific Causes of Gastrointestinal Bleeding

Age greater than 50

Chronic renal failure

Cutaneous hemangiomas

Chronic diarrhea/abdominal pain

Acquired-immunodeficiency syndrome (AIDS)

\section{Carcinoma}

Vascular ectasia/angiodysplasia

Blue rubber bleb nevus syndrome

Celiac sprue

Acquired-immunodeficiency syndrome (AIDS) 


\section{Table-II}

\section{Comparison of Gastrointestinal Investigative Techniques for Occult and Obscure Bleeding}

\section{Method}

Small bowel follow -

through/enteroclysis

Tagged red blood cell scan

Angiography

Push enteroscopy

Capsule endoscopy

Method

Double balloon enteroscopy

Single balloon enteroscopy

Endoscopic Ultrasou nd

CT scan

\section{Advantage}

Minimal side effects or risk

Good for rapid bleeding of $0.1-0.4 \mathrm{ml} / \mathrm{min}$

Good in young patients to find diverticulum

Good for rapid bleeding of

$>0.5 \mathrm{ml} / \mathrm{min}$

Can intervene in rapid

bleeding if source is located

Direct visualization and intervention

Allows examination of most of the small bowel

Noninvasive

Advantage

Direct visualiz ation and treatment of any part of small bowel lesion.

Rapid and eas ier to operate than double balloon

Allows full thickness guided biopsy of abnormal mucosa

Noninvasive

\section{Disadvantage}

Misses mucosal lesions Enteroclysis tube uncomfortable

Nonspecific, fa lse localizations, and missed bleeding Cannot determine cause

Specific only for Meckel's diverticulum

Invasive; risk of intestinal infarction with embolization Less likely to determine cause than endoscopy

Risk of intravenous contrast reaction

Invasive, endoscopic risk, patient discomfort, misses part of jejunum and ileum

No intervention capability Physician interpretation is time -consuming

Disadvantage

Invasive, endoscopic risk, patient discomfort, expensive, not widely available.

Not widely available

Not widely available

No intervention capability, expensive and low diagnostic yield
The British Society of Gastroenterology's guidelines propose small-bowel evaluation with capsule endoscopy as first test for patients with bleeding if no bleeding source is identified on upper and lower endoscopy, as diagnostic yield is highest during or soon after a bleeding episode 26,27 . On the basis of the findings, the clinician may proceed with push enteroscopy or double-balloon enteroscopy. Intraoperative enteroscopy should be reserved for patients with recurrent bleeding and transfusion dependency. 
So in Patients with OGIB if endoscopic evaluation of upper and lower tracts is negative or equivocal, $2^{\text {nd }}$ look examination by repeat upper and lower endoscopy is preferred before small bowel imaging [consensus/expert guidelines]. Cameron's erosions (within a hiatal hernia), peptic ulcer disease and vascular ectasias are the most common upper tract lesions found on repeat endoscopy, and cancer and angiodysplasias (Figure 8) are the most commonly overlooked lower tract abnormalities ${ }^{1}$.

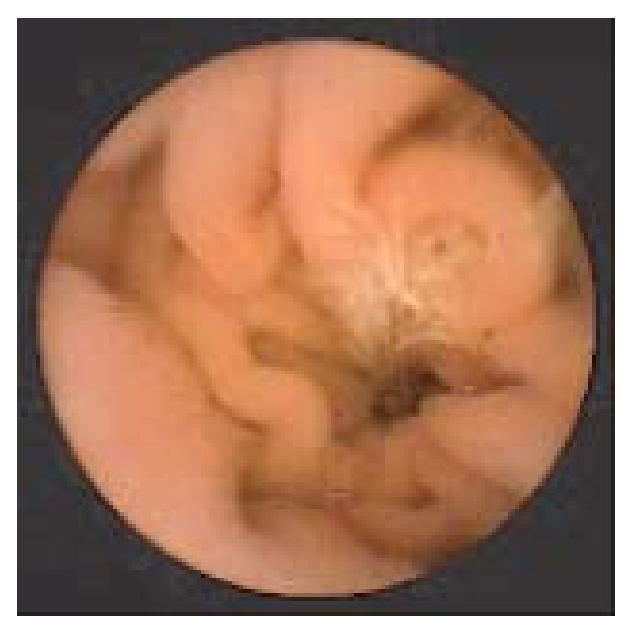

Fig.-8: Bleeding jejunal ulcer

Endoscopically lymphomas may present as enlarged gastric folds, mucosal nodularities, multiple polypoid masses with or without ulceration or with a diffuse infiltrative process. One unusual feature is that peristalsis is often preserved. Diagnosis can be difficult, sometimes requiring full thickness biopsy but when combined with endoscopic ultrasound (EUS) diagnostic accuracy approaches $100 \%$.

Haemobilia or Wirsungorrhagia into the duodenum may require the use of a side viewing endoscope to make the diagnosis. It has been suggested that the endoscopic appearance at the ampulla of a filiform clot suggests biliary bleeding and of fresh bleeding a pancreatic origin. Angiographic or CT findings may be needed to make the diagnosis of an aneurysm, pseudoaneurysm or arterio portal venous fistula.

A history of abdominal aortic graft surgery should prompt a careful endoscopic examination of the second and third parts of the duodenum. If aorto-oesophageal fistula is suspected, CT investigation should be undertaken prior to endoscopy in theatre since endoscopy can precipitate torrential bleeding.

\section{Management}

Importance of resuscitation can not be overemphasized. With adequate resuscitation as defined by hemodynamic stability there is significant reduction of post procedure complications. Antibiotic prophylaxis is another key component in the preparation prior to endoscopic intervention as there is likely development of transient bacteremia. Coagulation factor and platelet factor abnormalaties should be assesed and corrected prior to endoscopy. Appropriate level of sedation can be reached through use of a benzodiazepine combined with a narcotic.

Endoscopy plays a major role in the evaluation of OGIB and the management approach is summarized in the flowchart (Table III).

\section{Treatment and Outcome}

Treatment varies according to the etiology of bleeding, its severity and patient comorbidities. Treatment options include endoscopic, angiographic, pharmacotherapy, surgical therapies and non-specific measures. Endoscopic therapies include thermal contact probes, laser coagulation, injection sclerotherapy and banding.

Thermal ablation of bleeding is the treatment most commonly used for accessible lesions. Endoscopic therapy is successful in more than $90 \%$ of cases of Dieulafoy's bleeding. Adrenaline is frequently injected into the base prior to definitive treatment with electrocoagulation or more recently, band ligation. During angiography, interventional radiologists inject vasopressin or embolization material into bleeding vessels. Medical therapies are one of the few options available for diffuse vascular lesions, but they have limited success rates ${ }^{28}$. Various thermal coagulation devices, including heater probes, bipolar probes, the $\mathrm{Nd}$ :YAG laser and the argon plasma coagulator appear to be successful in treating these lesions. Coagulation should begin at the central feeding arteriole and work peripherally. Primary treatment modality is the bipolar probe because it causes more superficial injury than other thermal methods. Laser treatment can cause deep injury relatively easily and must be used carefully.

Complication rates are low for gastric lesions and in the small bowel. Colonic complications are reported in 
Table-III

Flowchart - Management plan of Obscure Bleeding

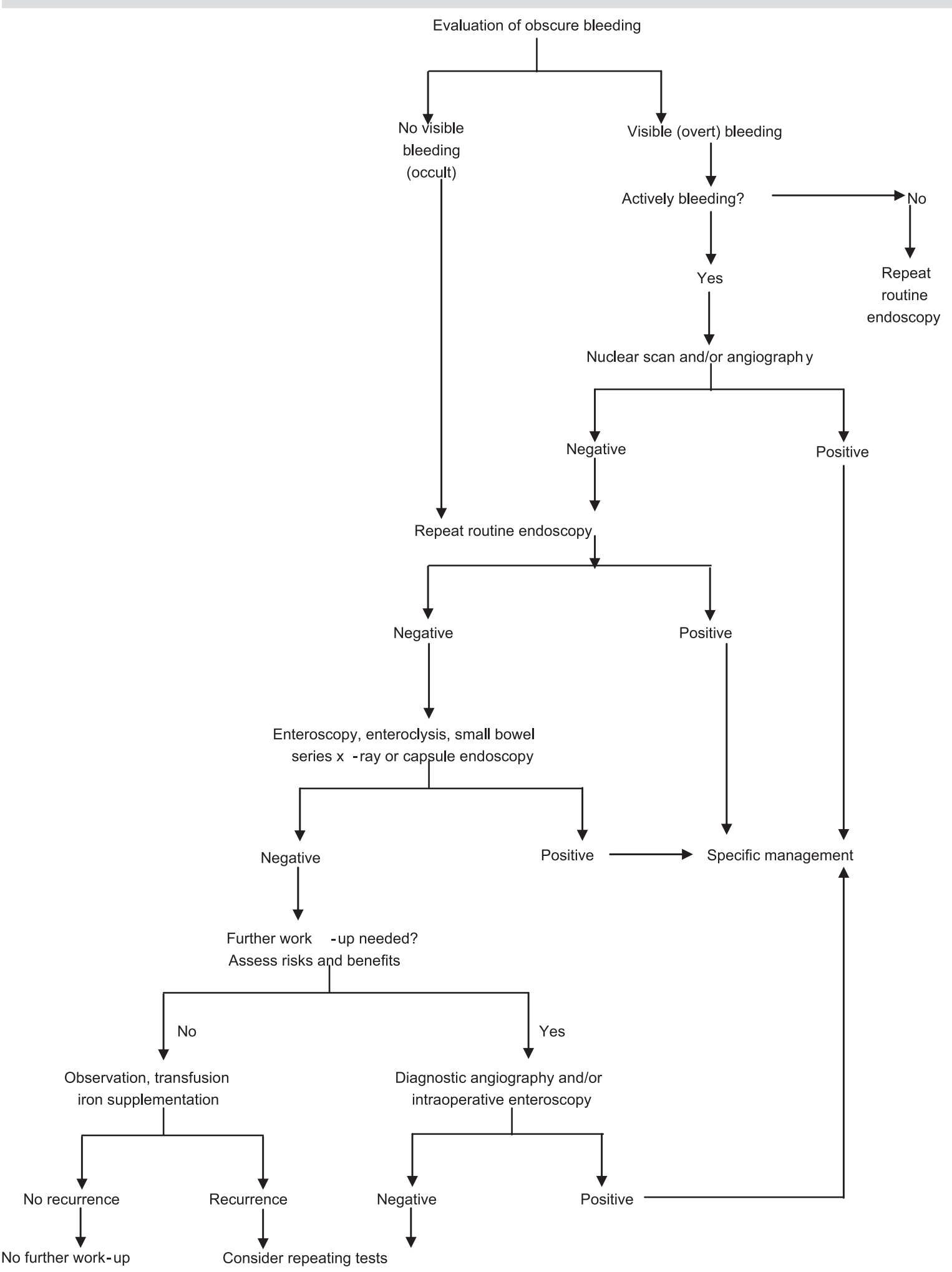


up to $10 \%$ cases and include partially treated lesions and perforation. Treatment is not required for incidental lesions. Treatment of isolated gastric lesions will often terminate bleeding, whereas many small bowel lesions are not reached and new lesions develop with time. Some patients will maintain a stable hemoglobin on iron therapy alone.

Gastrointestinal bleeding from arterial venous malformations has been successfully treated with combined hormone (ethinyl estradiol 0.035-0.05 mg; norethisterone $1 \mathrm{mg}$ ) therapy. However, continuous use of hormones for months has considerable side effects. The risk of thromboembolic events increases although observational studies have not confirmed a risk increase $^{29}$. With hereditary telangiectasias, von Willebrand's disease, or angiodysplasias in the setting of end-stage renal disease Octreotide (Sandostatin), given at a dosage of 0.05 to $0.1 \mathrm{mg}$ subcutaneously two to three times per day, has been successful in case studies.

Treatment of lymphoma is according to histology and includes helicobacter eradication for MALT lymphomas.

Unless a single causative lesion is identified, surgical therapy should be a last resort. Currently, exploratory laparatomy is seldom preferred without concomitant intraoperative enteroscopy in cases of transfusion dependant bleeding ${ }^{30}$. Patients with obscure bleeding often have multiple bleeding sites and bleeding may persist after surgery.

Non-specific therapy represents the primary approach to treatment in selected patients and should not be considered as failure of diagnostic approach. Nonspecific measures include iron replacement, correction of coagulation or platelet disorders, intermittent blood transfusions if anemia cannot be corrected with iron supplement alone. These measures are beneficial when rate of blood loss is slow and in elderly patients in whom the risk of further diagnostic evaluation is greater.

\section{Conclusion:}

There is no single efficient diagnostic test or therapeutic approach in the management of obscure GI bleeding. Most patients will benefit from a meticulous investigative routine that attempts to visualize as much of the bowel as necessary. Definitive therapy may not be possible in all the cases.
Non specific measures are beneficial when rate of blood loss is slow and in the elderly. There have indeed been a number of improvements in the management of OGIB but morbidity is still high. Adopting new technologies (endoscopic ultrasound, confocal laser endomicroscopy, narrow band imaging and endoscopic suturing devices) may be helpful in conquering this challenging problem.

\section{Acknowledgement:}

The authors thank Sabrina Mehnaz for her help in designing and editing the manuscript.

\section{References:}

1. Rockall TA, Logan RF, Devlin HB, Northfield TC. Incidence of and mortality from acute upper gastrointestinal haemorrhage in the United Kingdom. Steering Committee and members of the National Audit of Acute Upper Gastrointestinal Haemorrhage BMJ, 1995;311:222-226

2. Blatchford O, Davidson LA, Murray WR, Blatchford M, Pell J. Acute upper gastrointestinal haemorrhage in west of Scotland: case ascertainment study. BMJ, 1997;315:510-514

3. Zuckerman GR, Prakash C, Askin MP, et al. AGA technical review on the evaluation and management of occult and obscure gastrointestinal bleeding. Gastroenterology 1999;118:201-221.

4. Foutch PG. Angiodysplasia of gastrointestinal tract. Am J Gastroenterol 1993;88:807-818.

5. Lewis BS, Kornbluth A, Waye JD. Small bowel tumours: yield of enteroscopy . Gut 1991;32:763-765.

6. Kwo PY, Tremaine WJ. Nonsteroidal anti-inflammatory druginduced enteropathy: case discussion and review of the literature. Mayo Clin Proc. 1995;70:55-61.

7. Palmer K, Nairn M. Management Of Acute Gastrointestinal Blood Loss: Summary of SIGN Guidelines. BMJ, 2008;337:928-931.

8. Morris AJ, Mokhashi M, Straiton M, Murray L, Mackenzie JF. Push enteroscopy and heater probe therapy for small bowel bleeding.

Gastrointest Endosc 1996;44:394-397.

9. Jensen DM, Machicado GA. Bleeding Colonic Angioma: Endoscopic

coagulation and follow up. Gastroenterology 1985;88:1433

10. Chalasani N, Cotsonis G, Wilcox CM. Upper gastrointestinal bleeding in patients with chronic renal failure: role of vascular ectasia. Am J Gastroenterol 1996;91:2329-2332

11. Gostout CJ. Angiodysplasia and aortic valve disease: lets close the book on this association editorial; comment. Gastrointest Endosc 1995;42:491-493

12. Reilly HF, Al-Kawas FH. Dieulafoy's lesion. Diagnosis and management. Dig Dis Sci 1991;36:1702-170713 
13. Dieulafoy G. Exulceratio simplex. Bull Acad Med 1898:3940,49-82

14. Jabbari M, Cherry R, Lough JO, Daly DS, Kinnear DG, Goresky CA. Gastric antral vascular ectasia: the watermelon stomach. Gastroenterology 1984;87:1165-1170

15. Cameron AJ, Higgins JA. Linear gastric erosion. A lesion associated with large diaphragmatic hernia and chronic blood loss anemia. Gastroenterology 1986;91:338-342

16. Czerniak A, Thompson JN, Hemingway AP, Soreide O, Benjamin IS, Allison DJ. Hemobilia. A disease in evolution. Arch Surg 1988;123:718-721

17. Oksuzoglu BC, Oksuzoglu G, Cakir U, Bayir T, Esen M. Blue rubber bleb nevus syndrome. Am J Gastroenterol 1996;91:780-2.

18. Bennett ML, Sherertz EF, Jorizzo JL. Skin lesions associated with gastrointestinal diseases. In: Yamada T, Alpers DH. Atlas of gastroenterology. 3d ed. Philadelphia: Lippincott Williams \& Wilkins 2003:127-45.

19. Zaman A, Katon RM. Push enteroscopy for obscure gastrointestinal bleeding yields a high incidence of proximal lesions with in reach of a standard endoscope. Gastrointest Endosc 1998;47:372-376.

20. Chak A, Koehler MK, Sundaram SN, et al. Diagnostic and therapeutic impact of push enteroscopy: analysis of factors associated with positive findings. Gastrointest Endosc 1998;47:18-22.

21. Hayat M, Axon AT, O’Mahony S. Diagnostic yield and effect on clinical outcomes of push enteroscopy in suspected small bowel bleeding. Endoscopy 2000;32:369-372.
22. Descamps C, Schmit A, Van Gossum A. Missed upper gastrointestinal tract lesions may explain occult bleeding. Endoscopy 1999;31:452-455.

23 American Gastroenterological Association medical position statement: evaluation and management of occult and obscure gastrointestinal bleeding. Gastroenterology 2000;118:197201.

24. Bampton PA, Holloway RH. A prospective study of the gastroenterological causes of iron deficiency anaemia in general hospital. Aust NZ J Med 1996;26:793-799.

25. Kepczyk T, Kadakia SC. Prospective evaluation of gastrointestinal tract in patients with iron-deficiency anemia. Dig Dis Sci 1995;40:1283-1289.

26. Sidhu R, Saunders DS, Morris AJ, MC Alindon ME. Guidelines on small bowel enteroscopy and capsule endoscopy in adults. Gut 2008; 57:125-36.

27. Lewis BS, Swain P. Capsule endoscopy in the evaluation of patients with suspected small intestinal bleeding: results of a pilot study. Gastrointest Endosc 2002;56:349-53.

28. Van Cutsem E, Rutgeerts P, Vantrappen G. Treatment of bleeding gastrointestinal vascular malformations with oestrogen progesterone. Lancet 1990;335:953-955]

29. Lewis BS, Salomon P, Rivera-MacMurray S, Kornbluth AA, Wenger J, Waye JD. Does hormonal therapy have any benefit for bleeding angiodysplasia? J Clin Gastroenterol 1992;15:99-103.

30. Zukerman GR, Prakash C.Acute lower Intestinal bleeding:part II:etiology, therapy and outcomes. Gastrointest Endosc 1999;49:227-238. 